\title{
DYNAMICAL PROPERTIES OF GALACTIC OH/IR STARS
}

\author{
M. N. SEVENSTER, R. A. ARNOLD, L. O. SJOUWERMAN \\ Sterrewacht Leiden, \\ Postbus 9513, 2300 RA Leiden, The Netherlands
}

\section{Introduction}

An OH/IR star is an intermediate mass AGB star which loses mass and is optically obscured by its envelope. Such stars can be detected in the infrared and by radio maser lines. In particular the $\mathrm{OH}$ line $(1612 \mathrm{MHz})$ is bright and has a distinctive profile, that allows us to determine the radial velocity of the central star. OH/IR stars are excellent tracers for the study of Galactic dynamics because they are bright, unaffected by extinction in the $\mathrm{OH}$ line and therefore visible throughout the Galaxy - and because they represent a wide range of stellar masses. The outflow velocity of the circumstellar shell is most likely driven by radiation pressure from the central star on the dust. Therefore outflow velocity increases with stellar luminosity and metallicity, and decreases with age.

We have selected two samples of $\mathrm{OH} / \mathrm{IR}$ stars for our present study. The first, the Central sample, is a set of 149 stars from Lindqvist et al. (1992). The second sample consists of 700 stars selected from IRAS PSC and observed by te Lintel Hekkert et al. (1991), covering the Galactic bulge and the better part of the galactic disc.

\section{Kinematic properties}

There is clear evidence of galactic rotation in both samples. One of the primary objectives of this study is to construct a model which can account for the velocity distribution of the two sets. In figure 1 we show the correlation of the observed rotation - measured by the product $v_{c} \ell$ - and the circumstellar shell expansion velocity $v_{\text {exp }}$. We have ranked the stars in the two samples in order of increasing expansion velocity and plotted rank along the abscissa. The cumulative sum of $v_{c} \ell$ is plotted on the ordinate. If a star appears at upper right or lower left in figure 1(a) then $v_{c} \ell$ is positive 
- in which case it is rotating in a prograde sense - and in the other two quadrants the product $v_{c} \ell$ is negative. In both samples the set of stars with low expansion velocities does not have a strong rotation about the Galactic center, and the cumulate hovers near zero. At high expansion velocities however, the cumulate climbs steadily, indicating that it is primarily these stars which are responsible for the rotation seen in figure 1 . The outflow velocities are significantly higher among the Central sample stars than those in the IRAS sample. The transitions of behavior in figure 1 occur at about $17 \mathrm{~km} / \mathrm{s}$ for the Central stars and at $13.5 \mathrm{~km} / \mathrm{s}$ for the IRAS stars.
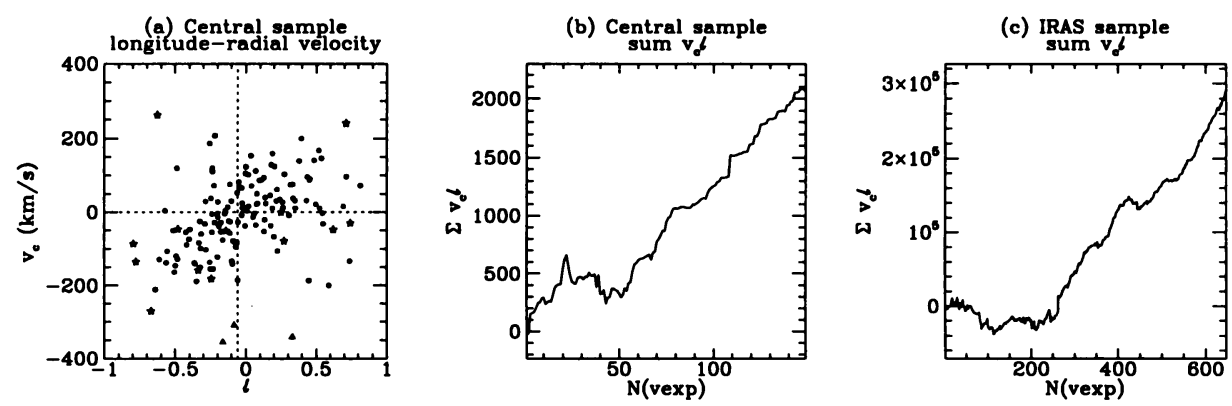

Figure 1. (a) Distribution of line of sight velocity $v_{c}$ and Galactic longitude $\ell$ for the Central sample. (b) Cumulate sum of $v_{c} \ell$ plotted against $v_{\text {exp }}$ rank for the Central sample and (c) for the IRAS sample.

Global dynamical distribution functions, derived from axisymmetric orbital components (Dejonghe 1989), show a compact, fast rotating circular disc in the Central sample. The IRAS sample contains an isotropic bulge distribution and a weak rotating component. In order to improve the kinematic constraints on the models we are extending the sample of $\mathrm{OH} / \mathrm{IR}$ stars in the region $|l|<45^{\circ}$ and $|b|<3^{\circ}$, and in addition measuring proper motions for stars near the center.

If stars in the center are more metal rich than stars of the same age elsewhere and if we assume that expansion velocity is primarily determined by metallicity, then these two samples could have similar ages. Alternatively, the central stars may be genuinely younger than those found in the IRAS sample, in which case one would also expect them to have higher metallicities and higher expansion velocities. Our dynamical results favor the latter scenario.

\section{References}

Dejonghe, H., 1989. AJ 343, 242

Lindqvist, M., Habing, H. J. \& Winnberg, A., 1992. A\&A 259, 118

te Lintel Hekkert,P., Habing,HJ., Caswell,J., Norris,R., Haynes,R. 1991. A\&AS 90, 327 